\title{
Influence of defects on the electrical and optical characteristics of blue light-emitting diodes based on III-V nitrides
}

\author{
I. Mártil, E. Redondo, and A. Ojeda \\ Departamento de Electricidad y Electrónica, Facultad de Ciencias Físicas, \\ Universidad Complutense de Madrid, 28040 Madrid, Spain
}

(Received 2 August 1996; accepted for publication 13 November 1996)

\begin{abstract}
We have measured the electrical and optical properties of blue light-emitting diodes (LEDs) based on III-V nitrides. The current-voltage characteristic is described by means of the relation $I=I_{0} \exp (\alpha V)$. In this equation $\alpha$ is temperature independent, suggesting a process of conduction by tunneling, as was recently reported also for blue-green LEDs based on III-V nitrides [Appl. Phys. Lett. 68, 2867 (1996)]. We explain the differences between blue and blue-green devices taking into account the tunneling process across semiconductor interfaces, in which a great number of defects is present. The light output intensity of the LED as a function of junction-voltage data reveals a dependence on the junction-voltage of the type $L=L_{0} \exp (q V / 1.4 \mathrm{KT})$, indicating that the radiative recombination path is via deep levels located at the forbidden gap. Furthermore, we find that the light output-current characteristic follows a power law like $L \propto I^{p}$. From the analysis of data it appears that, contrary to expectations, the nonradiative centers are saturated at very low current values that are comparable to the values at which this saturation takes place in LEDs based on III-V arsenides with a low content of defects. (C) 1997 American Institute of Physics. [S0021-8979(97)05304-8]
\end{abstract}

Blue light-emitting diodes (LEDs) based on III-V nitrides have recently shown high brightness characteristics ${ }^{1}$ in spite of the presence of an extremely high density of dislocations (in the $2-10 \times 10^{10} \mathrm{~cm}^{-2}$ range $^{2}$ ) that is several orders of magnitude higher than those measured in LEDs based on III-V arsenides and phosphides. Blue and blue-green devices are commercially available from Nichia Chemical Industries. ${ }^{1,3}$ The main difference between them is the composition of the active layer $\left(\operatorname{In}_{0.06} \mathrm{Ga}_{0.94} \mathrm{~N}\right.$ for blue devices and $\mathrm{In}_{0.23} \mathrm{Ga}_{0.77} \mathrm{~N}$ for the blue-green emitters).

Here, we present a study of the influence of defects on the transport properties and on the optical characteristics of the Nichia blue LEDs and we compare the results with those reported in blue-green LEDs from the same manufacturer. ${ }^{4}$

The blue LED analyzed in this study is device part No. NLPB500. The physical structure is composed of different layers, all of them grown by metalorganic chemical vapor deposition (MOCVD). Briefly, the structure is as follows: a $n$-GaN bottom contact layer $(\sim 4 \mu \mathrm{m})$, followed by a double heterostructure $(\mathrm{DH})$ composed of an $n-\mathrm{Al}_{0.15} \mathrm{Ga}_{0.85} \mathrm{~N}$ carrier confinement layer $(\sim 0.15 \mu \mathrm{m})$, an $\operatorname{In}_{0.06} \mathrm{Ga}_{0.94} \mathrm{~N}$ active layer, $\mathrm{Si}$ and $\mathrm{Zn}$ codoped $(0.05 \mu \mathrm{m})$, and a $p-\mathrm{Al}_{0.15} \mathrm{Ga}_{0.85} \mathrm{~N}$ carrier confinement layer $(0.15 \mu \mathrm{m})$. Last is a $p-\mathrm{GaN}$ top contact layer $(0.5 \mu \mathrm{m})$.

We have measured the current-voltage $(I-V)$ characteristic and the light output intensity as a function of junctionvoltage $(L-V)$ and LED output current $(L-I)$. The $I-V$ and $L-V$ data are influenced by the presence of a series resistance at high voltages. We have suppressed this influence following the procedure described in Ref. 5. Over the entire current range, where the influence of series resistance is present $\left(4 \times 10^{-4}-2 \times 10^{-2} \mathrm{~A}\right)$, the experimental data can be fitted to the equation

$$
I=I_{0} \exp \left[q\left(V-I R_{s}\right) / n K T\right],
$$

using a single value of the series resistance, $R_{s}$. This means that $R_{s}$ does not change as the operating current of the LED rises. Throughout the range of measurement, we observe no shunt resistance influence. In Eq. (1) all the symbols have the usual meaning.

In Fig. 1, we present the LED current as a function of junction-voltage, i.e., after the effect of $R_{s}$ was removed. Two different zones appear, depending on the range of the junction-voltage we consider. Both of them are described by the equation:

$$
I=I_{0} \exp (\alpha V),
$$

with

$$
\begin{aligned}
I_{0}= & (4 \pm 1) \times 10^{-19} \mathrm{~A}, \quad \alpha=12.5 \pm 0.2 \quad \mathrm{~V}^{-1} \\
& \text { for } V<2.6, \\
I_{0}= & (1 \pm 0.6) \times 10^{-17} \mathrm{~A}, \quad \alpha=11.2 \pm 0.3 \quad \mathrm{~V}^{-1}
\end{aligned}
$$

for $V>2.6$,

i.e., we can assess the presence of two different process of conduction. We made the measurements at temperatures ranging between 0 and $80{ }^{\circ} \mathrm{C}$. The slope of the characteristic in both zones was temperature independent. The reverse saturation current was slightly temperature dependent. 


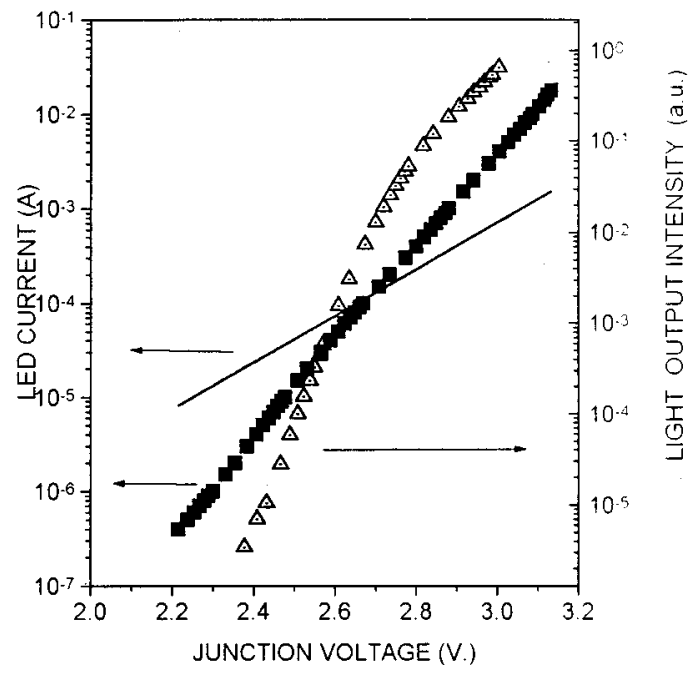

FIG. 1. LED current $(\square)$ and light output intensity $(\triangle)$ as a function of junction voltage for the blue LED. Also shown by the solid line is the $I-V$ characteristic measured in the Nichia blue-green LED, as described in Ref. $4\left[I=2.7 \times 10^{-11} \exp (5.7 \mathrm{eV})\right]$.

The values of $\alpha$ in Eq. (2) and the temperature independence of this parameter are typical features of a process of conduction by tunneling. This is in contrast with the $I-V$ characteristics of homojunction and heterojunction LEDs based on III-V arsenides and phosphides, ${ }^{6}$ in which both diffusion current and space charge recombination current are the main conduction processes.

The tunneling transport process across the interface of a semiconductor heterojunction is described by the equation: ${ }^{7}$

$$
I=B N_{T} \exp \left[4 / 3 \hbar\left(m^{*} \epsilon / N\right)^{1 / 2} V\right],
$$

where $B$ is a constant and $N_{T}$ is the density of states located in the forbidden gap at the interface or in the space charge zone that provides the tunneling path. The other symbols, $m^{*}, \epsilon$, and $N$, stand for the carrier effective mass, the electrical permitivity, and the doping, respectively, of the material of the higher gap in the $\mathrm{DH}$, which in this case is the $p-\mathrm{Al}_{0.15} \mathrm{Ga}_{0.85} \mathrm{~N}$ layer. ${ }^{4}$

Equation (3) describes the situation of a single-step tunneling recombination pathway. In heterojunctions, however the tunneling process might involve a more complex multistep pathway. As a consequence, Eq. (3) describes the conduction process in a qualitative way. We have compared our results with those recently reported by Casey et al. ${ }^{4}$ on $\mathrm{Ni}$ chia blue-green LEDs. We have included the $I-V$ characteristic of this diode in Fig. 1, which may be represented also by Eq. (3). This characteristic was also explained by Casey et al. as tunneling dominated conduction.

Some insight into the conduction process of the devices was provided by Casey et al. by taking into account the defective nature of the constituent layers of the DH. However, the differences between blue and blue-green devices may be understood by considering the lattice mismatch between the active layer and the confining layers of the devices. The lattice mistmatch between the active and the confining layers in the blue emitter is about $1 \%$, and about $3 \%$ in the blue-green LED. Hence, at the InGaN/AlGaN interface mistmatch dis-

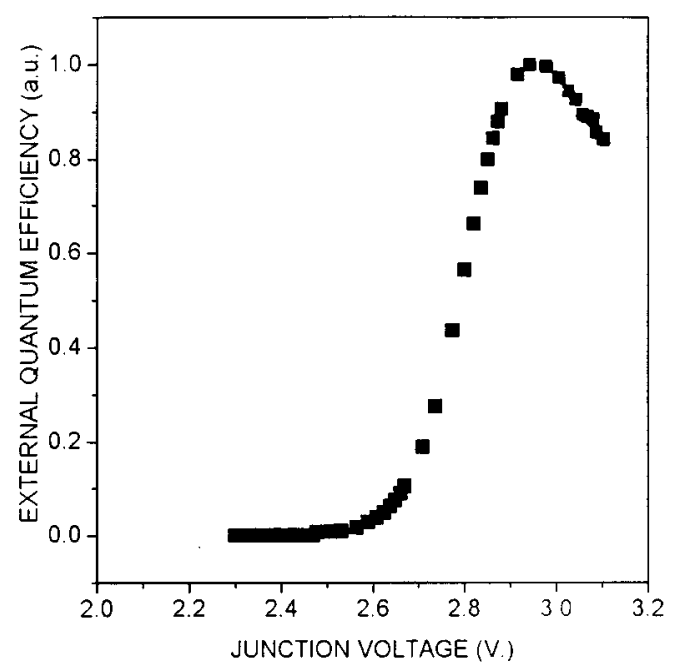

FIG. 2. External quantum efficiency of the blue emitter as a function of junction voltage.

locations should appear. The number of these dislocations should be greather in the blue-green diode than in the blue LED due to the higher mistmatch for the first device. As a consequence, the interface of the blue-green LED should be more defective than that of the blue LED. ${ }^{8}$ Hence, tunneling processes are expected to show higher saturation current in the device that presents the higher lattice mistmatch, i.e., in the blue-green emitter. The data for the reverse saturation current, shown in Ref. 4 for the blue-green LED, $2.7 \times 10^{-11}$ $\mathrm{A}$, is higher than the values measured by us, $4 \times 10^{-19}$ (low voltages) $-10^{-17} \mathrm{~A}$ (high voltages) in the blue LED. This is in agreement with the qualitative features of Eq. (3), in which the parameter $N_{T}$ is mainly responsible for the value of the reverse saturation current. The comparison we present here for blue and blue-green LEDs indicates that the presence of lattice mistmatch should be taken into account to

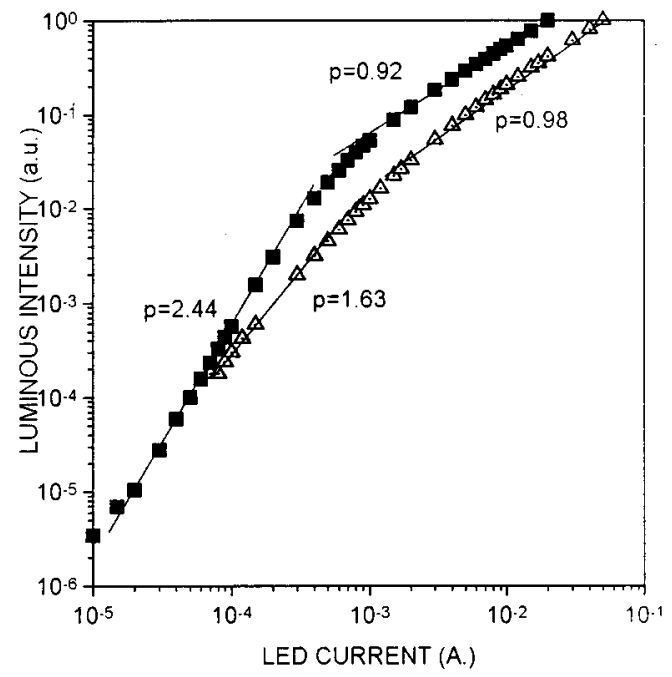

FIG. 3. Light output intensity as a function of LED current for the blue LED $(\boldsymbol{\square})$ and for a high efficiency infrared LED based on GaAs $(\triangle)$. We also indicate the values of $p$ in Eq. (5) for the two devices in the superlinear and linear zones. 
elucidate definitively the mechanism of conduction in these devices.

Turning to the light emission characteristics of the blue LEDs, we have plotted the output light intensity as a function of junction-voltage in Fig. 1 . Notice that the $L-V$ and $I-V$ dependences are very different.

The $L-V$ data, for voltages below $2.8 \mathrm{~V}$ (or currents lower than $3 \times 10^{-4} \mathrm{~A}$ ), can be fitted to the equation:

$$
L=L_{0} \exp (q V / n K T)
$$

where the $n$ factor turns out to be $1.4 \pm 0.1$. This value suggests that the major radiative recombination pathway is via deep levels. There are two possible radiative recombination pathways in Nichia blue LEDs: ${ }^{9,10}$ shallow donor to Zn deep acceptor or deep donor to $\mathrm{Zn}$ deep acceptor. The deep donor, of unknown origin, is located $\sim 200 \mathrm{meV}$ below the shallow donor. Both donors can be represented by Eq. (4), although the corresponding value for $n$ may be different. The data of Fig. 1 do not provide enough information for us to decide between the two different possibilities. The most relevant aspect of Fig. 1 is that the dependences exhibited by the $I-V$ and $L-V$ characteristics with junction-voltage are completely different. This means that only a small fraction of the operating LED current is effectively converted to photons. In other words, almost all the LED current is nonradiative.

The $L-V$ data deviate from Eq. (4) at junction voltages above $2.8 \mathrm{~V}$, indicating the filling of $\mathrm{Zn}$ acceptors as the current (or the junction voltage) rises. This is more clearly observed in Fig. 2 where we plot the external quantum efficiency of the device as a function of junction voltage. The maximum for this parameter is reached at $V=2.9 \mathrm{~V}$; at higher junction voltages, the relative quantum efficiency falls down since band-to-band radiative recombination processes are dominant. Therefore, it is not surprising that the $L-V$ curve levels off for junction voltages above $\sim 2.8 \mathrm{~V}$.

In Fig. 3, we present the $L-I$ characteristic of the blue LED together with the same data for a high efficiency commercial infrared LED based on GaAs. The results can be fitted with the power law 6

$$
L \propto I^{P},
$$

where $p$ accounts for the influence of defects in the characteristics of light emission. For the sake of clarity in the following, we will label the different regimes as superlinear, if $p>1$, and linear, if $p \sim 1$. In the two devices, a superlinear zone (at low currents) and a linear zone (at high current), appear with the $p$ values indicated in Fig. 3. The superlinear zone is directly related to the presence of nonradiative centers that provide a shunt path to the current. When these centers are saturated, the linear relation holds. Since the blue LED has a density of defects much higher than the LED based on GaAs, the value of $p$ in the superlinear zone is greater for the blue LED (2.44) than for the LED of GaAs (1.63).
The striking aspect that Fig. 3 reveals is that, in both devices, the saturation of the nonradiative centers is reached at almost identical currents $(\sim 1-3 \mathrm{~mA})$, while the dislocation density differs and is about $10^{10}$ and $10^{4} \mathrm{~cm}^{-2}$ for the blue and the infrared LEDs, respectively. This result is apparently in conflict with the values of $p$ quoted in Fig. 3. In fact, with such a high value of $p$ for the blue LED, it would be expected to reach the saturation of nonradiative centers at currents much higher than those experimentally measured in this device. This result could indicate that not all the defects are nonradiative in nature. Otherwise, it seems difficult to achieve a level of saturation similar to that, measured in LEDs based on III-V arsenides. Lester et al. ${ }^{2}$ proposed recently that the nature of the defects present in LEDs based on nitrides could be different than in LEDs based on arsenides. The ionic character of the bonding in the nitrides may be responsible for such anomalous behavior. However, the data in Fig. 3 do not allow us to further clarify this particular point. More work on this issue seems to be necessary for a definitive answer.

Summarizing, we have studied the influence of defects in the electrical and optical characteristics of the blue LED (NLPB500). The analysis of the $I-V$ characteristic indicates that the main conduction process is a tunneling mechanism, with two different zones depending on the junction voltage. This behavior is qualitatively similar to that reported for blue-green LEDs. The main difference, which is the reverse saturation current, may be explained in terms of the mismatch between the different layers of the DH. The $L-V$ characterization shows that the main radiative recombination pathway is via deep levels in the forbidden gap, and that only a small fraction of the total current contributes to the light emission.

The $L-I$ characteristic points out that nonradiative centers are saturated at operating currents similar to those measured in high efficiency LEDs based on GaAs, in spite of the great difference in the content of defects between them. This surprising result points toward a different nature of defects in III-V nitride LEDs in comparison with their counterparts, III-V arsenides, and allows one to attain high brightness characteristics in blue LEDs at the usual operation currents.

${ }^{1}$ S. Nakamura, T. Mukai, and M. Senoh, Appl. Phys. Lett. 64, 1687 (1994).

${ }^{2}$ S. D. Lester, F. A. Ponce, M. G. Craford, and D. A. Steigerwald, Appl. Phys. Lett. 66, 1249 (1995).

${ }^{3}$ S. Nakamura, T. Mukai, and M. Senoh, J. Appl. Phys. 76, 8189 (1994).

${ }^{4}$ H. C. Casey, Jr., J. Muth, S. Hrishnamkutty, and J. M. Zauada, Appl. Phys. Lett. 68, 2867 (1996).

${ }^{5}$ D. Fuch and H. Sigmund, Solid-State Electron. 29, 791 (1986).

${ }^{6}$ M. G. Craford and F. M. Steranka, Encyclop. Appl. Phys. 8, 485 (1994).

${ }^{7}$ A. G. Milnes and D. L. Feucht, Heterojunctions and MetalSemiconductor Junctions (Academic, New York, 1973), p. 42.

${ }^{8}$ S. Nakamura, Microelectron. J. 25, 651 (1994).

${ }^{9}$ W. E. Carlos, E. R. Glaser, T. A. Kennedy, and S. Nakamura, Appl. Phys. Lett. 67, 2376 (1995).

${ }^{10}$ W. E. Carlos, E. R. Glaser, T. A. Kennedy, and S. Nakamura J. Electron. Mater. 25, 851 (1996) 